International Journal of Engineering \& Technology, $7(2.25)(2018) 109-112$
International Journal of Engineering \& Technology
Website: www.sciencepubco.com/index.php/IJET
Research paper

\title{
Segmentation of exudates to assess diabetic retinopathy by reni's entropy based thresholding
}

\author{
Josline Elsa Joseph $^{1}$ *, R. J. Hemalatha ${ }^{1}$, Bincy Babu ${ }^{1}$, T. R. Thamizhvani ${ }^{1}$, A. Josephin Arockia Dhivya ${ }^{1}$, \\ Sangeethapriya.K $\mathbf{K}^{1}$ \\ ${ }^{1}$ Department of Biomedical Engineering,Vels Institute of Science, Technology\& Advanced studies, Pallavaram, Chennai-600117, \\ India \\ *Corresponding author E-mail: joslinejoseph02@gmail.com
}

\begin{abstract}
Objective: Diabetic retinopathy is a critical pathological disease condition which affects the lives of millions of people everyday. Exudates found in the eye are one of the important signs of Diabetic retinopathy. This work aims to segment exudates for faster detection and treatment of Diabetic retinopathy.Methods: This paper proposes a robust and efficient method to segment exu-dates. Initial pre-processing work applies adaptive unsharp masking which sharps the areas based on the level of smoothness in the image preventing accentuation of noise. Optic disc is removed by active contour model. The exudates are then segmented by Renyi's Entropy based thresholding which choses the optimal threshold for segmentation, exploiting Renyi's entropy da-ta.Results: The performance of the proposed system was evaluated and found better than state of art results giving accuracy, sensitivity and specificity $94.5 \%, 95.1 \%$ and $96.2 \%$ respectively.Conclusion: Effective computer aided system is essential for accurate exudates detection. The proposed algorithm utilises the advantages of adaptive unsharp masking in medical image pro-cessing along with Renyi's entropy based thresholding to detect Exudates, which performs better than traditional thresholding techniques.
\end{abstract}

Keywords: Lung; Feature Extraction; Detection; Pulmonary Embolism

\section{Introduction}

Diabetic retinopathy is one among the several chronic diseases of the eyes causing irreversible damage to eye. The chances of being affected with retinopathy rises in diabetes mellitus affected population [1]. Early screening of exudates in the eye can promote faster treatment. Hence, CAD system is helpful especially for camp screenings and also to help physicians to confirm the several stages of diabetic retinopathy. [2-4]

There are vast researches continuing in the field of diabetic retinopathy diagnosis. Region growing algorithm and it variations for exudate detection are done in colour retinal images [5-6]. Thresholding methods including grey level thresholding was applied by authors D.Kavitha et al[7] and a combination of multi-level thresholding with region growing is adopted in digital retinal images by D.Usher et al[8]. Benefits of clustering methods namely $\mathrm{k}$ means clustering, fuzzy c means clustering and improvises have been adopted for detection of exudates in diabetic retinopathy affected eye images [9-11]. Different Morphological operations have also been employed performed for exudates extraction [12-13] along with various classifiers. A technique of multi-space clustering method is employed by authors R. Annunziata et al [14]. One of the disadvantages of the existing system is over segmentation in morphological based techniques. The proposed methodology overcomes the disadvantages of the existing system by the application of Renyi's entropy for exudate detection which utilizes the spatial information in the image. Hence the proposed system outruns the existing methods due to Renyi's specific and suitable threshold selection based thresholding of exudates.

\section{Materials and methods}

The images used in this work were taken from DIARETDB1 [15]. It consists of 89 images captured under the field of view of 50 degrees consisting of [5] normal images and 84 mild diabetic retinopathy images. In this paper, robust and improved algorithm for exudates segmentation for diabetic retinopathy screening is proposed in which Exudates are extracted by Renyi's Entropy based Thresholding. The initial work includes pre-processing the image. The green channel of the original image was extracted as it better displays the fine details of the eye. In the next step, adaptive unsharp masking filter was applied to the image which depicts the region of interest with higher effectiveness. The next step of pre-processing included optic disc elimination which is essential as its intensity and colour appear similar with exudates. Optic disc was eliminated by Active contour method which was followed by exudates segmentation. The block diagram of the proposed work is given in figure 1. 


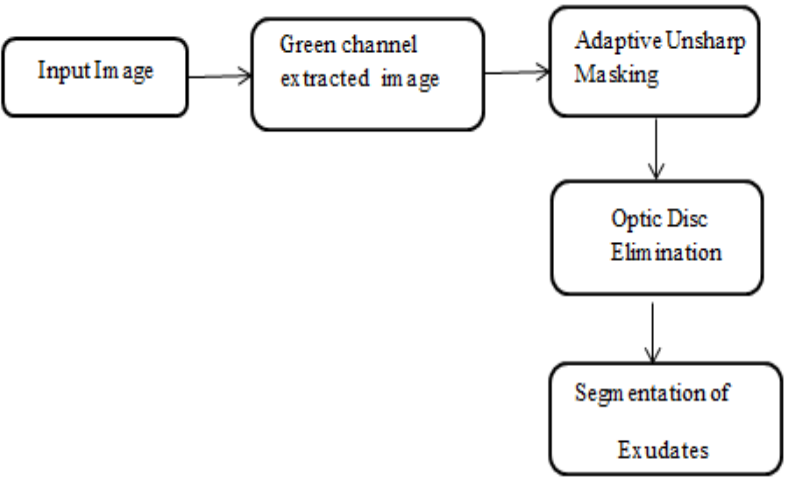

Fig. 1: Block Diagram of the Proposed System.

\section{Preprocessing}

\subsection{Adaptive unsharp masking}

The green channel image is filtered using adaptive unsharp masking [16]. The traditional unsharp masking removes the low frequency components in the image and projects the edge features and improves the contrast. It works like a high pass filter where the contrast can be adjusted based on the user input value. The adaptive unsharp masking filter introduces a filter other than functions carried out by high pass filter, which decisively measures the regional local dynamics of the input image. The improved algorithm sets two thresholds $\mathrm{t} 1$ or $\mathrm{t} 2$ depending on whether the input is a smoothed image or medium or high contrast image. The traditional unsharp mask filter equation is given below in Eq (1)

$\mathrm{U}(\mathrm{a}, \mathrm{b})=\mathrm{I}(\mathrm{a}, \mathrm{b})+\pi \mathrm{H}(\mathrm{a}, \mathrm{b})$

$\mathrm{U}(\mathrm{a}, \mathrm{b})$ represents the output image

$\mathrm{I}(\mathrm{a}, \mathrm{b})$ is the input image \& $\mathrm{H}(\mathrm{a}, \mathrm{b})$ shows the low frequency removed image after corrections by $\pi$ scale value. An adaptive filter introduced in the above system only enhances the medium contrast areas, avoiding smooth areas to prevent inclusion of noise projection. The improved Scaling value in the adaptive filter works based on the difference in the actual and founded dynamic value of the image examined.

\subsection{Optic disc elimination}

Optic Disc is seen as yellow - white luminous component in both normal and abnormal retinal image. Exudates also exhibit higher contrast and brightness similar to optic disc. Hence to accurately detect exudates, it is essential to remove optic disc from the image. Active contour Model [17] was chosen to effectively extract the optic disc. It captures the exact geometry of the region of interest. The user input provides initial boundary point which is continued by image forces that discover the region to be extracted. A prior knowledge of the region of interest gives better contour segmentation results. The initial selection of active contour determines the accurate detection of the entire boundary of the target. Active contour model works by changing its shape and position from the initial contour towards the minimal energy state. Also called as Snake model, it moves itself through the domain of the retinal image and settles towards the local minimum energy state.

The forces in Snake model are a combination of external and internal forces and also the energy of the image under consideration aiming to reduce the total energy to the least level. The Total Energy of Active snake model is a summation of three types of Energy namely (i) Internal Energy (EI) that depends on the degree of the spline equating the shape of the region of interest. (ii) External Energy (EE) that involvesall the external forces which are user dependant and the other energy factors. (iii) Energy of the retinal image under consideration (ER) which gives out the vital data about the illumination of the spline representing the target object.

$$
\mathrm{ET}=\mathrm{EI}+\mathrm{EE}+\mathrm{ER}
$$

The optic disc of the input retinal image is removed by using the geometric information and intensities by the active contour model which improves the success rate of candidate extraction.

\subsection{Exudates segmentation}

Exudates in the image are segmented using Renyi's Entropy based Thresholding. Entropy can be explained as the quantitative measure of diverseness or randomness in a set of data for its description which was introduced by Shannon [18]. In Renyi's entropy based thresholding for image manipulation $[19,20]$, the spatial information of the pixels are also considered for optimal threshold selection.

Consider an image A of size $\mathrm{Mx} N$ with $\mathrm{L}$ grey levels where $\mathrm{f}(\mathrm{j}, \mathrm{k})$ is the grey level of the pixel at the spatial location $(\mathrm{j}, \mathrm{k})$.In the image, two probability distributions are derived from the grey level distribution for object and background of the image thereby separating them as two classes $\mathrm{X}$ and $\mathrm{Y}$ given as in equations $3 \& 4$ where p0,p1..p255 represents the probabilities of grey level distribution.

$$
\begin{aligned}
& X: \frac{P 0}{p(X)} \quad \frac{P 1}{p(X)} \quad \frac{P_{3_{3}}}{p(X)} \quad \\
& Y: \frac{P t+1}{p(Y)} \frac{P t+2.8(4)}{p(Y)} \quad \frac{P 255}{p(Y)} \\
& \text { Where, } p(X)=\sum_{i=0}^{t} p^{\text {ind }} \quad p(Y)=\sum_{i=t+1}^{255}(\dot{p)}
\end{aligned}
$$

Where, and (5)

The a priori Renyi's entropy of order, for the image is given in $\mathrm{Eq}$ (6) where $\neq 1$ and the total entropies for both object and background classes gives the Renyi's entropy for thresholding. (6)

$\eta_{t}=\frac{1}{1-\alpha} \operatorname{In} \sum_{k=0}^{255}(p k)^{\eta}$

The value of helps in optimal selection of threshold value resulting. Once the exudates are detected, the remaining borders are cleared by opening operation to avoid any false candidate pixels.

\section{Results}

The results of red, green, blue colour channels and multi-channel composite image and their respective histograms are given in figure 2.This analysis provides the most suitable colour channel for further processing. The benefits of adaptive unsharp masking are exploited in retinal pre-processing giving enhanced appearance of the bright exudates in the image. The pre-processed images are given in figure 3 . Reni's entropy is a useful data for thresholding in image processing which gives good segmentation results of exudates. The exudates detected image is given in figure 4 .

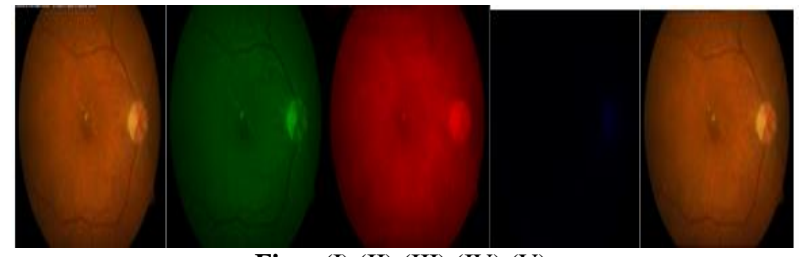

Fig. : (I) (II) (III) (IV) (V).

(A)

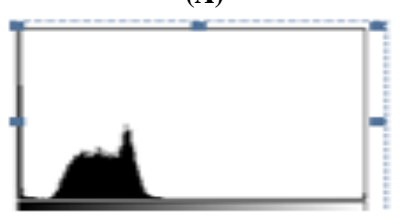

(B) 


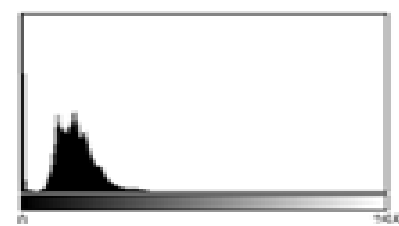

(C)

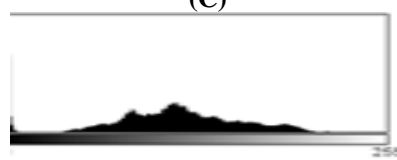

(D)

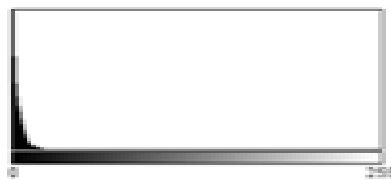

(E)

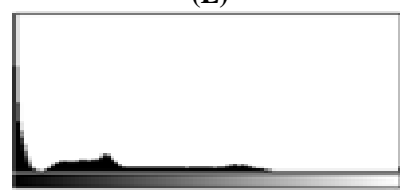

Fig. 2: Evaluation of Primary Color in Retinal Image (I) Input Image (Ii) Green Channel Image (Iii) Red Channel Image (IV) Blue Channel Image (V) Multi-Channel Composite Image (VI)-(X) Histogram of the Above Images Respectively.

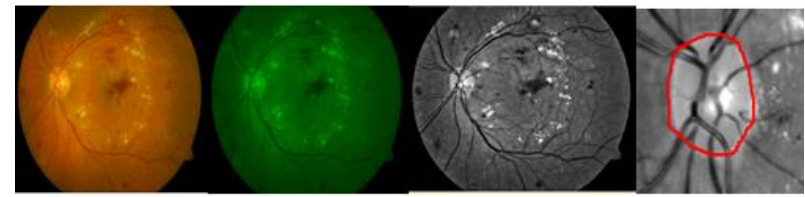

Fig. : (I) (II) (III) (IV).0.

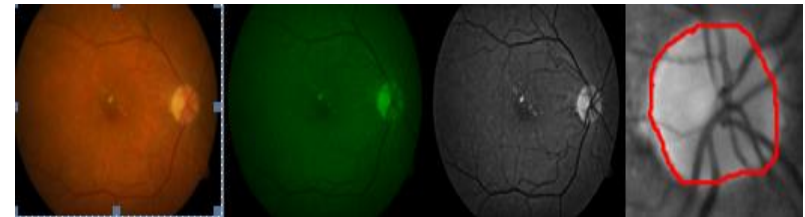

Fig. 3:.Pre-Processed Images (I) \& (V) Input Images, (II) \& (VI) Green Channel Extracted Images (III) \& (VII) Adaptive Unsharp Masking Images, (IV)\&(VIII) Active Contour Based Optic Disc Elimination.
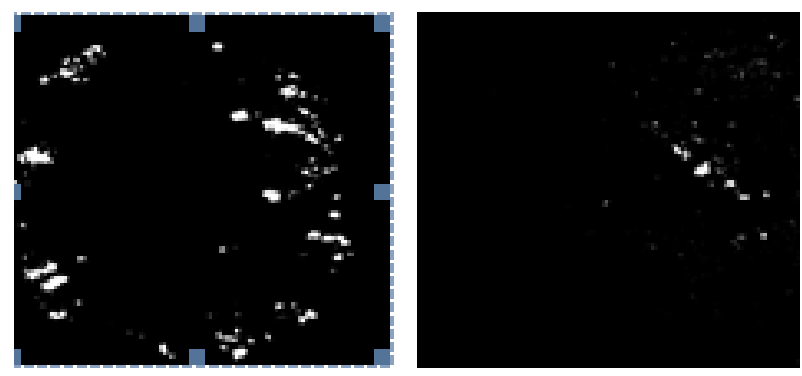

Fig. 4: Exudates Segmentation by Renyi’s Entropy Thresholding.

\section{Discussion}

The normal and diabetic retinopathy images are checked for their appearance and histogram examined in the red, blue and green channels. The green channel image depicts maximum features of the image. The three primary colours were differentiated to understand the pathological features that are most prominent in these channels. The blue channel image and histogram depicts minimal information. Red and green channel extracted images convey major retinal features in which red channel contains maximum information. Retinal Vessels are poorly envisioned in red channel but the optic disc channel is projected with high contrast. Green channel image and the histogram convey exudates presence better than red channel extracted image. The adaptive unsharp masking provides corrected signal by varied adaptive filter which enhances the areas optimally based on certain criteria and avoids attenuation of noise and artefacts. Hence the resultant image is depicts the region of interest more precisely. Active contour model applied for removal of optic disc exploits the geometric information and also utilises the image forces to achieve the target object. The advantage of Renyi's Entropy based thresholding is the specific selection of the most suitable value for thresholding. Performance Evaluation measures like accuracy sensitivity and specificity were estimated for the proposed work. Sensitivity: evaluates the precision of the proposed system to correctly segment exudates.

Accuracy: defines the overall correctness of the proposed system. Specificity: evaluates the correctness of the system to detect background pixels.

The Proposed work provided good accuracy, sensitivity and specificity values of $94.5 \%, 95.1 \%$ and $96.2 \%$ respectively.

\section{Conclusion}

Exudates detection is considered one major tool for diagnosing this disease. Our work proposes application of Renyi's entropy based thresholding for exudates segmentation in the retinal fundus image. The three colour channels and their histograms are analysed before choosing green channel image as input for further processing. Active contour model works well for elimination of optic disc and followed by exudates segmentation and removing borders by opening operation. The performance evaluation measures provide higher accuracy, sensitivity and accuracy results than state of art methods. Future work will address issues of feature selection and it is ranking for screening diabetic retinopathy.

\section{References}

[1] L. Giancardo, F. Meriaudeau, T. P. Karnowski, Y. Li, S. Garg, K.W.Tobinand E. Chaum, 'Exudates based Detection In Fundus Images Using Publicly Available Datasets', Med Image Ana., vol 16,no.1,pg 216-226,2012..

[2] C. Agurto, V. Murray, H. Yu, J.Wigdahl, M.Pattichis, S.Nemeth et al., 'A Multiscale Optimization Approach To Detect Exudates In The Macula', IEEE Journal of Biomedical and Health Informatics, vol.18,no.4,pg.13228-1336,2014.

[3] A. F. Aqeel and S. Ganesan, 'Automated Algorithm For Retinal Images And Drusens Detection, 'Segmentation And Measurement', In Proceedings Of The IEEE International Conference On Electro Information Technology, pg 206-215, 2014.

[4] P.M Rokade and R. R. Manza, 'Automatic Detection Of Hard Exudates In Retinal Images Using Haar WAVELET Transform,' International Journal Of Application Or Innovation In Engineering \&Management, Vol .4,No.5, pg 402-410,2015.

[5] H. Li, O. Chutatape, 'Automated Feature Extraction in Color Fundus Retinal Images by a Model Based Approach IEEE Transactions on Biomedical Engineering. Vol 51, No.2, pg 264-254, 2004.

[6] B.M. Ege, L. Hejlese, O. V. Larsen, B. Moller, M. Kerr, 'Screening for Diabetic Retinopathy Using Computer Based Image Analysis And Statistical Classification', Computer Methods Programs Biomed, Vol 63, no.3,pg 165-175.2000.

[7] D. Kavitha and S.S. Devi, 'Automatic Detection Of Optic Disc And Exudates In Retinal Images', IEEE International Conference On Intelligent Sensing and Information Processing, pg.501-506,2005.

[8] D. Usher, M. Dumskyj, M Himaga, T. H. Williamson, S. Nussey, J. Boyce, 'Automated Detection of Diabetic Retinopathy in Digital Retinal Images: A Tool for Diabetic Retinopathy Screening.' Diabetes Med.pg. 84-90.2004.

[9] A. Somasundaram and J. Prabhu, 'Detection of Exudates for the Diagnosis of Diabetic Retinopathy', International Journal of Innovation and Applied Studies, Vol.3, No.1, pg 116-120, 2013.

[10] A. Osareh, M. Mirmehdi, B. Thomas, R. Markham, 'Automated Identification Of Diabetic Retinal Exudates In Retinal Exudates In Digital Color Images, Br.J. Ophthamol.,Pg 1220-1223,2003.

[11] Dunn, J.C, 'A Fuzzy Relative Of The ISODATA Process And Its Use In Detecting Compact Well Separated Clusters,Cybernetics And Systemspg 32-57,1973. 
[12] A. Sopharak, B. Uyyanonvarab, S. Barman, T. H. Williamson. 'Automatic Detection Of Diabetic Retinopathy Exudates From Non Dilated Morphology Methods, Computerized Medical Imaging And Graphics, Pg 720-727, 2008.

[13] C .E .Hann, M. Narbot, M.Macaskill, 'Diabetic Retinopathy Detection Using Geometrical Techniques Related to the Underlying Pathology. Int. Conf. Image Vis.Comput,pg1-8,2010.

[14] R. Annunziata, A. Garzelli, L. Ballerini, A. Mecocci And E. Trucco, 'Leveraging Multiscale Hessian-Based Enhancement With A Novel Exudate Inpainting Technique For Retinal Vessel Segmentation, - IEEE Journal Of Biomedical And Health Informatics, Vol.20,No.4,Pg 1129-1138,2016.

[15] TomiKauppi, Kalensnykiene V, Kamarainen J-K, Lensu L, Sorri, I, Raninen A, R. Voutilanien, Uusitalo, H.Kalviainen,J. Pietila, DIARETDB1 Diabetic Retinopathy Database And Evaluation Protocol,Online: http:// www.it.lut.fi/project/imageret/diaretdb1.

[16] Andrea Poesel, Giovanni Ramponi and V.John Mathews, 'Image Enhancement Via Adaptive Unsharp Masking', IEEE Transactions on Image Processing, Vol 9, No.3, Pg 505-510,200 0.

[17] S.Weeratunga and C. Kamath, 'An Investigation Of Implicit Active Contours For Scientific Image Segmentation' ,In Proceedings of SPIE, vol 5308,pg 210-221,2004.

[18] J. Lin, 'Divergence Measures Based on the Shannon Entropy', IEEE Trans. Inf Theory, Vol.37 (1), pg 145-151, 1991.

[19] Prasanna Sahoo, Carrye Wilkins and Jerry Yeager, Threshold Selection Using Renyi's Entropy Thresholding', Pattern Recognition, Elsevier, Vol.30, No.1, pg 71-84, 1997.

[20] P.K.S and A.C.K.W.J.N .Kapur, ' A New Method For Gray Level Picture Thresholding Using The Entropy Of The Histogram,Computer Vision Graphic,Image Process,Vol.29,Pg 273-285,1985. 